\title{
Calibration method for rotating-analyzer ellipsometers
}

\author{
J. M. M. de Nijs, A. H. M. Holtslag, A. Hoeksta, and A. van Silfhout \\ Faculty of Applied Physics, Twente University, P.O. Box 217, 7500 AE Enschede, The Netherlands
}

Received January 8, 1988; accepted May 8, 1988

\begin{abstract}
In operating a rotating-analyzer ellipsometer one must know the plane of incidence accurately. We present a new calibration method, phase calibration, which is complementary to residue calibration Phase calibration is shown to be superior to the residue method for $\Delta<\pi / 6$ or $\Delta>5 \pi / 6$.
\end{abstract}

\section{INTRODUCTION}

One of the main sources of errors in ellipsometry is inaccurate calibration; if the plane of incidence is not known correctly, all azimuthal angles of the optical components involved, such as the polarizer, the analyzer, and sometimes the compensator, contain errors. Thus calibration errors proliferate, leading ultimately to errors in the measured quantities $\Delta$ and $\Psi$. Not withstanding the fact that the final errors in $\Delta$ and $\Psi$ can be eliminated in first order in a twozone measurement, 1,2 it is obvious that an accurate method of calibration is desirable.

With the introduction of the rotating-analyzer ellipsometer (REA), an appropriate measuring technique for this type of ellipsometer was developed. ${ }^{3-5}$ First, the plane of incidence is determined by a technique that we shall call residue calibration, a method similar to the calibration methods used earlier in nulling ellipsometers. ${ }^{6}$ Second, once the plane of incidence is known, the signal-detection system can be calibrated. Finally, $\Delta$ and $\Psi$ can be obtained from a single measurement and the previously determined calibration parameters. In this way, the complete measurement consists of two parts, a calibration and the actual measurement. That other methods are possible as well was shown recently by Kawabata, who presented a method for a RAE to measure $\Delta$ and $\Psi$ without previous calibration (a kind of an integral method). ${ }^{7}$ In Kawabata's method, four quantities are measured, all of them with a finite accuracy, from which $\Delta$ and $\Psi$ as well as the calibration parameters are obtained. Obviously, errors can cause a strong mixing of these four quantities, and one should be alert to statistical and systematic errors. The first method has the advantage that, in principle, statistical errors are eliminated from the calibration and thus do not contribute to the errors in the two measured quantities, $\Delta$ and $\Psi$.

In practice it has been shown that residue calibration works well on metals in general. However, when applied to semiconductors in the visible-wavelength region or to nonabsorbing substrates, residue calibration is less useful, yielding poor results. In what follows we show that residue calibration becomes highly inaccurate with $\Delta$ approaching either 0 or $\pi$ rad. Under these circumstances, one could perform the calibration on another sample or at another wavelength; however, a complementary method for a direct approach should be desirable. In this paper we present such a complementary method, which looks much like residue calibration and which we call phase calibration. ${ }^{8}$

This paper is organized as follows. In Section 2 we present a general description of the measuring technique and the data handling used in the RAE. In Section 3 we treat residue calibration, for which we derive an expression offering the opportunity to calculate the accuracy of the calibration directly from the calibration itself. Phase calibration is presented in Section 4. It is shown that the two methods are complementary; phase calibration is most suited for cases of $\Delta$ near 0 or $\pi \mathrm{rad}$, and residue calibration for $\Delta$ near $\pi / 2$. The paper concludes with a short discussion on the work presented. An example is included, clearly illustrating the usefulness of each method under specific circumstances.

\section{GENERAL}

Concerning the RAE, we must distinguish the optical signal detected by the detector $\left(I_{\mathrm{opt}}\right)$ from the electrical signal $\left(I_{\mathrm{el}}\right)$, which is measured and finally evaluated by the controlling computer. Figure 1 shows the optical part of the RAE; the light flux passing the analyzer thus becomes

$$
\begin{aligned}
I_{\mathrm{opt}}(t)= & \frac{I_{0}\left|\tilde{r}_{p}\right|^{2}}{\left(8 \sin ^{2} \Psi\right)}\left\{\left[1-\cos 2\left(P-P_{\mathrm{c}}\right) \cos 2 \Psi\right]\right. \\
& +\left[\cos 2\left(P-P_{\mathrm{c}}\right)-\cos 2 \Psi\right] \cos 2\left(\omega_{A} t-A_{\mathrm{c}}\right) \\
& \left.+\left[\sin 2\left(P-P_{c}\right) \sin 2 \Psi \cos \Delta\right] \sin 2\left(\omega_{A} t-A_{c}\right)\right\},
\end{aligned}
$$

with $P$ and $\omega_{A} t=A$, respectively, denoting the polarizer and the analyzer azimuths relative to the plane of incidence, $P_{c}$ and $A_{c}$ the polarizer and analyzer readings corresponding to the plane of incidence, $I_{o}$ the light flux from the source, and $\left|\tilde{r}_{p}\right|$ the magnitude of the parallel complex reflection coefficient of the surface. Subsequently, the optical signal is transformed into an electrical one by the detector and passes a filter, reducing the noise but inherently introducing a frequency-dependent phase shift $\varphi$ and an amplitude attenuation $\eta$ : 


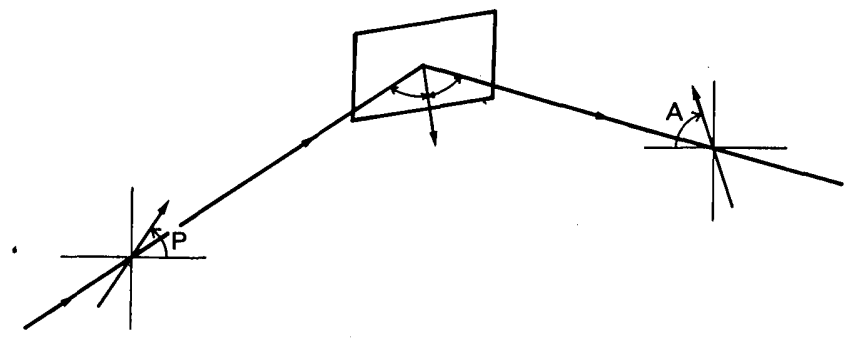

Fig. 1. Schematic diagram of the RAE ellipsometer showing the configuration of the polarizer, the sample, and the (rotating) analyzer. The azimuths of the polarizer and analyzer are measured relative to the plane of incidence.

$$
\begin{aligned}
I_{\mathrm{el}}(t)= & \frac{I_{0}\left|\tilde{r}_{p}\right|^{2}}{\left(8 \sin ^{2} \Psi\right)}\left\{\left[1-\cos 2\left(P-P_{c}\right) \cos 2 \Psi\right]\right. \\
& +\eta\left[\cos 2\left(P-P_{c}\right)-\cos 2 \Psi\right] \cos 2\left(\omega_{A} t-A_{c}-\varphi / 2\right) \\
& \left.+\eta\left[\sin 2\left(P-P_{c}\right) \sin 2 \Psi \cos \Delta\right] \sin 2\left(\omega_{A} t-A_{c}-\varphi / 2\right)\right\} .
\end{aligned}
$$

If we divide this expression by its average we find the more familiar, theoretical expression:

$$
\begin{aligned}
I_{\mathrm{el}}^{\mathrm{th}}(t)=1+\eta \alpha \cos 2\left(\omega_{A} t\right. & \left.-A_{c}-\varphi / 2\right) \\
& +\eta \beta \sin 2\left(\omega_{A} t-A_{c}-\varphi / 2\right),
\end{aligned}
$$

$\alpha=\left[\cos 2\left(P-P_{c}\right)-\cos 2 \dot{\Psi}\right] /\left[1-\cos 2\left(P-P_{c}\right) \cos 2 \Psi\right]$,

$\beta=\sin 2\left(P-P_{c}\right) \sin 2 \Psi \cos \Delta /\left[1-\cos 2\left(P-P_{c}\right) \cos 2 \Psi\right]$.

We have added the superscript th to denote where we study the detected signal from the more theoretical point of view. Note that the azimuth $A_{c}$ is not distinguished from the phase retardation $\varphi$; they can be summed and treated as a single unknown parameter, $2 A_{c}+\varphi$.

The RAE samples the signal $I_{\mathrm{el}}(t)$ during a number of cycles at the time points $t_{i}$, yielding the set of data points $\left\{I_{\mathrm{el}}\left(t_{i}\right)\right\}$. Subsequently the average $\langle I\rangle$ and the Fourier coefficients $a^{\prime}$ and $b^{\prime}$ are numerically calculated from the set $\left\{I_{\mathrm{el}}\right\}$ :

$$
\begin{aligned}
\langle I\rangle & =\sum_{i} I_{\mathrm{el}}\left(t_{i}\right), \\
a^{\prime} & =\sum_{i} I_{\mathrm{el}}\left(t_{i}\right) \cos \left(2 \omega_{A} t_{i}\right), \\
b^{\prime} & =\sum_{i} I_{\mathrm{el}}\left(t_{i}\right) \sin \left(2 \omega_{A} t_{i}\right) .
\end{aligned}
$$

Introducing the normalized Fourier coefficients $\alpha^{\prime}=a^{\prime} /\langle I\rangle$ and $\beta^{\prime}=b^{\prime} /\langle I\rangle$, we can construct an experimental expression $I_{\mathrm{el}}{ }^{\mathrm{ex}}(t)$ that corresponds to its theoretical analog $I_{\mathrm{el}}{ }^{\mathrm{th}}(t)$ [Eq. (3)]:

$$
I_{\mathrm{el}}^{\mathrm{ex}}(t)=1+\alpha^{\prime} \cos \left(2 \omega_{A} t\right)+\beta^{\prime} \sin \left(2 \omega_{A} t\right) .
$$

It should be emphasized that $\alpha^{\prime}$ and $\beta^{\prime}$ are the only quantities actually measured.
Equations (3) and (5) obviously have to be identical. Studying these equations, we notice that they contain five unknown quantities, $\Delta, \Psi, P_{c}, \eta$, and the sum $\left(2 A_{c}+\varphi\right)$, while only two quantities, the Fourier coefficients $\alpha^{\prime}$ and $\beta^{\prime}$, are obtained by a simple measurement. The calibration now has to yield the quantities $P_{c}, \eta$, and $\left(2 A_{c}+\varphi\right)$, enabling one to calculate $\Delta$ and $\Psi$ from one measured $\left(\alpha^{\prime}, \beta^{\prime}\right)$ pair. The values of $\eta$ and $2 A_{c}+\varphi$ are easily obtained once the plane of incidence is known. ${ }^{3,4}$ The problem is to obtain $P_{c}$ when one is able to measure only raw Fourier coefficients $\alpha^{\prime}$ and $\beta^{\prime}$. Now let us first rewrite Eqs. (3) and (5) for $I_{\mathrm{el}}^{\text {th }}$ and $I_{\mathrm{el}}{ }^{\mathrm{ex}}$ into more easily comparable forms:

$I_{\mathrm{el}}^{\mathrm{ex}}(t)=1+\left(\alpha^{2}+\beta^{\prime 2}\right)^{1 / 2} \cos \left(2 \omega_{A} t-\theta^{\prime}\right)$,

and

$$
\theta^{\prime}=\arctan \left(\beta^{\prime} / \alpha^{\prime}\right)
$$

$$
\begin{aligned}
I_{\mathrm{el}}^{\mathrm{th}}(t)=1+\eta\left(\alpha^{2}+\beta^{2}\right)^{1 / 2} \cos \left(2 \omega_{A} t-\theta\right), \\
\quad \theta=\arctan (\beta / \alpha)+2 A_{c}+\varphi .
\end{aligned}
$$

The equality of both now requires the independent equality of the prefactors and the arguments of the cosine. For a good understanding one should realize that, although $P_{c}, \eta$, and $2 A_{c}+\varphi$ are unknown, this prefactor and the argument survive the optic-electric and the Fourier transformation surprisingly well; apart from the constants $\eta$ and $2 A_{c}+\varphi$, they can be regarded as constants surviving all signal manipulations. From the equality of the prefactors, now, the residue method is derived. From the second requirement, the equality of the arguments of the cosine, another calibration method, phase calibration, can be derived.

\section{RESIDUE CALIBRATION}

According to the Aspnes method, ${ }^{3}$ one has to measure the residues $R$ as a function of the polarizer angle $P$; the residue is defined by

$$
\begin{aligned}
R & =1-\left(\alpha^{\prime 2}+\beta^{2}\right) \\
& \equiv 1-\eta^{2}\left(\alpha^{2}+\beta^{2}\right) .
\end{aligned}
$$

On substitution of the theoretical expressions for $\alpha$ and $\beta$ and with some subsequent manipulations, a compact expression for the residue can be obtained:

$$
R\left(P, P_{c}\right)=1-\eta^{2}+\left\{\eta \frac{\sin (\Delta) \sin (2 \Psi) \sin \left[2\left(P-P_{c}\right)\right]}{1-\cos (2 \Psi) \cos \left[2\left(P-P_{c}\right)\right]}\right\}^{2} .
$$

A look at this expression shows that it is independent of $2 A_{c}$ $+\varphi$, while $\eta$ is solely a constant that does not change the characteristic features of $R\left(P, P_{c}\right)$; the plane of incidence can be identified with the polarizer angle $P$ at which the minimum of $R$ is found. In the vicinity of $P_{c}$ the residue can be approximated by a parabolic behavior:

$$
\begin{aligned}
R\left(P, P_{c}\right)=1-\eta^{2}+\left[\eta \frac{2 \sin (\Delta) \sin (2 \Psi)}{1-\cos (2 \Psi)}\right]^{2} & \left(P-P_{c}\right)^{2}, \\
& \left|P-P_{c}\right| \ll 1 .
\end{aligned}
$$

This feature is used in the experimental technique; one measures the residue $R$ at a number of equidistant $P$ angles near $P_{c}$, where the parabolic behavior is expected. For $\Delta$ near $\pi / 2$ 
the curvature will be large, enabling one to determine the minimum of the parabola with a high degree of accuracy. However, as can be seen from Eq. (10), this minimum blurs with diminishing $\Delta$, finally obstructing the calibration for small $\Delta$ or $\Delta$ near $\pi$ rad. In what follows we present a quantitative treatment of this problem, from which we obtain some preliminary criteria for the application of the residue method.

As we have said, at a number $(N)$ of equidistant polarizer angles $P_{i}$ the residues $R_{i}$ are measured, constituting the set $S$ $=\left\{P_{i}, R_{i}\right\}$. Subsequently, the minimum is obtained by fitting a parabola

$$
R(P)=C_{0}+C_{1} P+C_{2} P^{2}
$$

to the set $S$. The fitting can be accomplished by means of a least-squares fitting procedure for parabolas, for which explicit formulas do exist $t^{4}$ :

$$
\begin{aligned}
C_{0}= & (N D)^{-1} \sum_{i=1}^{N}\left\{\left(p_{2} p_{4}-p_{3}^{2}\right)+\left(p_{2} p_{3}-p_{1} p_{4}\right) P_{i}\right. \\
& \left.+\left(p_{1} p_{3}-p_{2}{ }^{2}\right) P_{i}^{2}\right\} R_{i}, \\
C_{1}= & (N D)^{-1} \sum_{i=1}^{N}\left\{\left(p_{2} p_{3}-p_{1} p_{4}\right)+\left(p_{0} p_{4}-p_{2}{ }^{2}\right) P_{i}\right. \\
& \left.+\left(p_{1} p_{2}-p_{0} p_{3}\right) P_{i}^{2}\right\} R_{i}, \\
C_{2}= & (N D)^{-1} \sum_{i=1}^{N}\left\{\left(p_{1} p_{3}-p_{2}^{2}\right)+\left(p_{1} p_{2}-p_{0} p_{3}\right) P_{i}\right. \\
& \left.+\left(p_{0} p_{2}-p_{1}^{2}\right) P_{i}^{2}\right\} R_{i},
\end{aligned}
$$

with

$$
p_{k}=N^{-1} \sum_{i=1}^{N} p_{i}^{k}
$$

and

$$
D=p_{0} p_{2} p_{4}+2 p_{1} p_{2} p_{3}-p_{2}^{3}-p_{0}{p_{3}}^{2}-p_{1}^{2} p_{4}
$$

The minimum of the parabola [Eq. (11)] now is found at

$$
P_{c}=-C_{1} /\left(2 C_{2}\right)
$$

Next we direct our attention to the accuracy with which $P_{c}$ is obtained; we can derive a coarse criterion for the application of the residue method from it. Statistical errors do occur in any residue of the set $S$ that; ultimately, cause a random error in $P_{c}$. Systematic errors are present, too; e.g., optical activity of polarizers and windows will probably also shift $P_{c}{ }^{3} \quad$ However, these problems are beyond the scope of the present paper. Ambient light, as long as it is unpolarized, and the detector dark current have no influence on the determination of the plane of incidence because they only increase the unmodulated part in a $P$-independent way. This could cause a slight raising or lowering of the total residue curve, but the actual shape of the curve and the position of its minimum are not affected.

Let us assume that we have not chosen $P$ too far from $P_{c}$, so we may be assured of the proper parabolic behavior of the residue without any third- or higher-order contributions to it. In that case all residues $R_{i}$ lie along the parabola but are randomly distributed. Their standard deviation $\sigma_{R}$ can be estimated from

$$
\sigma_{R}^{2}=N^{-1} \sum_{i=1}^{N}\left(C_{0}+C_{1} P_{i}+C_{2} P_{i}^{2}-R_{i}\right)^{2} .
$$

The polarizer is rotated by some mechanical means, for which reason we assume random errors in the settings of $P_{i}$ to be negligible. Thus random errors in the coefficients $C_{0}$, $C_{1}$, and $C_{2}$ are introduced solely by the finite accuracy with which the residues are measured. Now, if we call the standard deviation of the individual residues $\sigma_{R}$, the standard deviations $\sigma_{0}, \sigma_{1}$, and $\sigma_{2}$ of the coefficients $C_{0}, C_{1}$, and $C_{2}$ can be derived from Eqs. (12):

$$
\begin{aligned}
\sigma_{0}^{2}= & \left(\frac{\sigma_{R}}{N D}\right)^{2} \sum_{i=1}^{N}\left\{\left(p_{2} p_{4}-p_{3}^{2}\right)+\left(p_{2} p_{3}-p_{1} p_{4}\right) P_{i}\right. \\
& \left.+\left(p_{1} p_{3}-p_{2}^{2}\right) P_{i}^{2}\right\}^{2} \\
\sigma_{1}^{2}= & \left(\frac{\sigma_{R}}{N D}\right)^{2} \sum_{i=1}^{N}\left\{\left(p_{2} p_{3}-p_{1} p_{4}\right)+\left(p_{0} p_{4}-p_{2}^{2}\right) P_{i}\right. \\
& \left.+\left(p_{1} p_{2}-p_{0} p_{3}\right) P_{i}^{2}\right\}^{2}, \\
\sigma_{2}^{2}= & \left(\frac{\sigma_{R}}{N D}\right)^{2} \sum_{i=1}^{N}\left\{\left(p_{1} p_{3}-p_{2}^{2}\right)+\left(p_{1} p_{2}-p_{0} p_{3}\right) P_{i}\right. \\
& \left.+\left(p_{0} p_{2}-p_{1}^{2}\right) P_{i}^{2}\right\}^{2},
\end{aligned}
$$

and, when we use Eq. (13), the standard deviation $\sigma_{P}$ of the azimuth $P_{c}$ becomes

$$
\sigma_{P}^{2}=\left(\frac{1}{2 C_{2}} \sigma_{1}\right)^{2}+\left(\frac{C_{1}}{2 C_{2}^{2}} \sigma_{2}\right)^{2} .
$$

The first term contributing to $\sigma_{P}$ is the main one; the second term may well be omitted for $C_{1} \equiv 0$ in the present situation. The expression states that the uncertainty in $P_{c}$ increases with decreasing curvature $C_{2}$ of the parabola or that the residue method becomes less accurate with $\Delta$ approaching 0

\section{Delta [deg]}

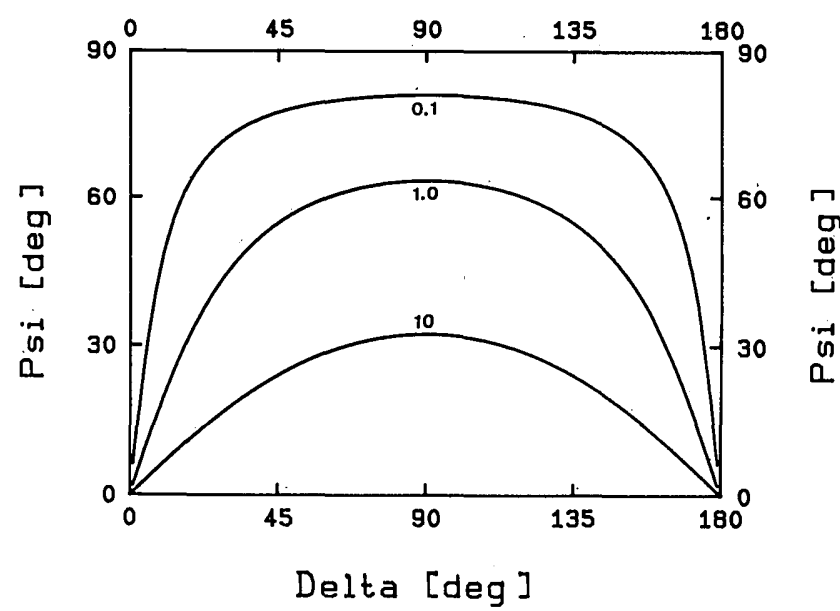

Fig. 2. Curves of constant curvature $C_{2}$ of the residue depicted in the $(\Delta, \Psi)$ plane. For $C_{2}<1$ the residue calibration becomes highly inaccurate. 


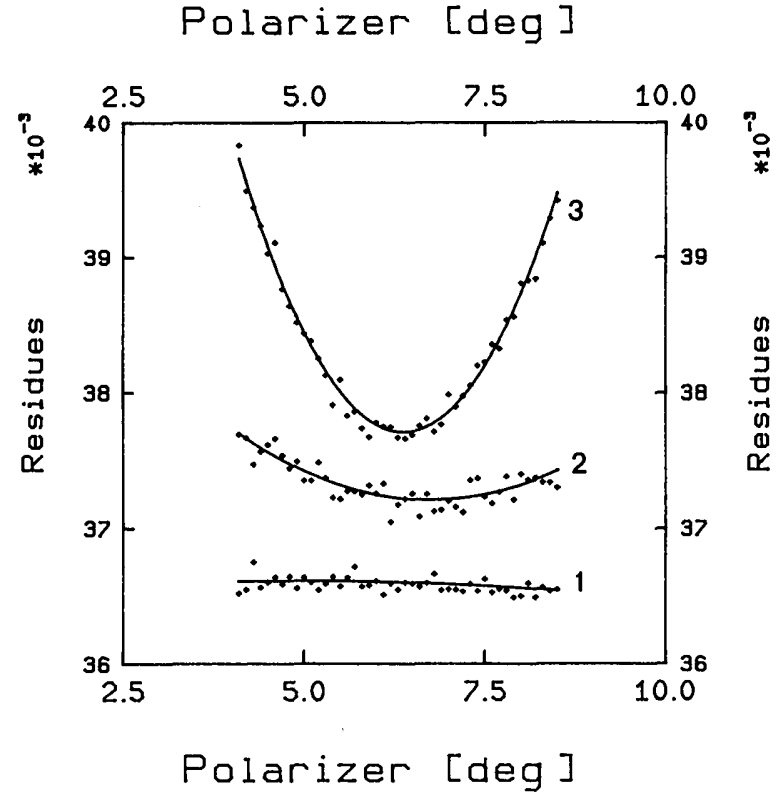

Fig. 3. Three typical examples of residue calibration, where we have plotted the residues and the best fits as functions of the polarizer azimuth $P$. The curves are shifted upward or downward to permit them to be distinguished better. Calibrations 1 and 2 are performed on the same clean $c$-Si sample, calibration 3 on a stainless-steel sample. The figure shows clearly that the method is less suitable for cases 1 and 2 than for case 3 . More information on these calibrations can be found in Table 1 .

Table 1. Calibrations Performed on the Same Samples and under Exactly the Same Circumstances Using the Residue Technique and the Phase Technique $^{a}$

\begin{tabular}{lrcc}
\hline & \multicolumn{3}{c}{ Calibration } \\
\cline { 2 - 4 } & 1 & 2 & 3 \\
\hline Sample & $c-\mathrm{Si}$ & $c-\mathrm{Si}$ & $\mathrm{M}$ alloy \\
Wavelength (nm) & 545 & 365 & 365 \\
Delta (deg) & 170 & 158 & 87 \\
Psi (deg) & 16 & 28 & 32 \\
Residue calibration & & & \\
$\quad P_{c}$ & 4.86 & 6.74 & 6.37 \\
$\quad \sigma_{P}$ & 7 & 1.0 & 0.1 \\
Phase calibration & & & \\
$\quad P_{c}$ & 6.36 & 6.42 & 12.6 \\
\hline
\end{tabular}

${ }^{a}$ Relevant information on the samples and the results obtained are given. The calibration angle $P_{c}$ must be near $6.36 \mathrm{deg}$. It can be seen that phase calibration is preferred for the $\mathrm{Si}$ sample ( $\Delta$ near $\pi$ ), while residue calibration must be used in case of the stainless-steel sample $(\Delta$ near $\pi / 2)$. The data correspond with Figs. 3 and 4.

or $\pi$ rad. In Fig. 2 we have plotted the curves of constant curvature $C_{2}$ in the $(\Delta, \Psi)$ plane, calculated from Eq. (10) with $\eta=1$, clearly showing the behavior expected. The minimum curvature at which the residue method can still be used can be estimated as follows. The polarizer angle $P$ should not be chosen out of the range of $0.10 \mathrm{rad}$; otherwise higher-order terms perturb the parabolic behavior of the residue. A typical accuracy $\sigma_{R}$ for a residue is $10^{-4}$, and consequently the increase of the residue should be of the order of $10^{-3}$ at the edge of the interval in which $P$ is chosen, to permit a good fitting of the parabola. In this way, one obtains $C_{2}=10$ as a coarse criterion for the applicability of the residue method. The corresponding region in the $(\Delta, \Psi)$ plane can be found in Fig. 2.

Note that the estimation presented above is a rough one, and probably the residue method can be used up to $C_{2}=1$. Formally, it is required that a criterion be derived for the application of the residue method from Eq. (16). The reason why we have deviated from this approach is that it is impossible to obtain proper estimations for $\sigma_{1}$ and $\sigma_{2}$, which must be obtained from the Eqs. (15). However, Eqs. (14-16) are easily implemented on the controlling computer, thus offering a check on the accuracy of the calibration, directly obtained from the calibration itself.

Some typical examples of the residue calibration are shown in Fig. 3. A summary of the results of these calibrations can be found in Table 1. The three calibrations presented are all performed on the same ellipsometer with a 70deg angle of incidence. Calibrations 1 and 2 are performed on a clean $c$-Si sample ( $\Delta$ near $\pi \mathrm{rad}$ ) at $\lambda_{1}=545 \mathrm{~nm}$ and $\lambda_{2}=$ $365 \mathrm{~nm}$, respectively. Without any changes in the alignment, we have calibrated a stainless-steel sample (calibration $3, \Delta \approx \pi / 2$ ) at $\lambda_{3}=365 \mathrm{~nm}$. In Fig. 3 and Table 1 it is shown clearly that calibration 1 is useless, calibration 3 is a good one, while calibration 2 should not be trusted too much.

As is shown, the residue method becomes less useful with $\Delta$ approaching 0 or $\pi \mathrm{rad}$, and for that situation a complementary method is desired. In Section 4 we present such a method, phase calibration, which is as easy to implement as the residue method on the controlling microcomputer.

\section{PHASE CALIBRATION}

Phase calibration is derived from the equality of the phases in Eqs. (6) and (7):

$$
\begin{aligned}
\theta^{\prime} & =\arctan \left(\beta^{\prime} / \alpha^{\prime}\right) \\
& \equiv \arctan (\beta / \alpha)+2 A_{c}+\varphi .
\end{aligned}
$$

Making use of Eqs. (3b) and (3c), we obtain

$$
\theta^{\prime}=\arctan \left[\frac{\sin 2 \Psi \sin 2\left(P-P_{c}\right) \cos \Delta}{\cos 2\left(P-P_{c}\right)-\cos 2 \Psi}\right]+2 A_{c}+\varphi .
$$

This phase, $\theta^{\prime}$, does not show any particular feature, mainly because of the unknown term $2 A_{c}+\varphi$. By measuring $\theta^{\prime}$ at $P$ and $P+\pi / 2$ and subtracting them, we obtain the phase difference $\Phi$ :

$$
\Phi(P)=\theta^{\prime}(P+\pi / 2),
$$

from which $2 A_{c}+\varphi$ is eliminated:

$$
\begin{aligned}
\tan [\Phi(P)] & =\frac{-\sin 2\left(P-P_{c}\right) \sin 4 \Psi \cos \Delta}{\sin ^{2} 2 \Psi-\left(1-\sin ^{2} 2 \Psi \cos ^{2} \Delta\right) \sin ^{2} 2\left(P-P_{c}\right)}, \\
& =\frac{K \sin 2\left(P-P_{c}\right)}{L+M \sin ^{2} 2\left(P-P_{c}\right)} .
\end{aligned}
$$

We have introduced $K(\Delta, \Psi), L(\Delta, \Psi)$, and $M(\Delta, \Psi)$ to stress the dependence of $\tan \Phi$ on $P-P_{c}$. The plane of incidence is obtained directly from the zero point of $\tan \Phi$ at $P=P_{c}$. In practice one has to measure tan $\Phi$ at a number of different 
azimuths $P, P$ chosen near $P_{c}$, yielding a function linear in $P$, which is shown from the first-order approximation of Eqs. (20):

$$
\Phi(P) \cong \frac{-4 \cos \Delta \cos 2 \Psi}{\sin 2 \Psi}\left(P-P_{c}\right), \quad\left|P-P_{c}\right| \ll 1 .
$$

The plane of incidence is then to be identified with the azimuth $P$ at which $\Phi(P)$ has its zero point. The advantage of this method shows when the method is applied on a surface with small or even zero $\Delta$; in these cases phase calibration yields good results, while residue calibration becomes much less accurate.

Apart from calibrating on the zero point of relation (21), another option exists: Eq. (20b) exhibits an extremum at $P-P_{c}=\pi / 4$. This extremum can be found by measuring $\tan \Phi$ as a function of the polarizer azimuth $P$ near $P=\pi / 4$. Subsequently its position is found by fitting a parabola to the measured values of $\tan \Phi$.

Phase calibration requires, as we have said, the measurement of the phase difference $\Phi(P)$ at a number $(N)$ of equidistant polarizer azimuths $P$ in the vicinity of $P_{c}$, yielding the set $\left\{P_{i}, \Phi_{i}\right\}$. Figure 4 is a typical example of such a measurement on a clean $c$-Si surface. This time, a linearregression method is used to determine the zero point from the set of data points. If we denote the straight line that has to be fitted to the $\operatorname{set}\left\{P_{i}, \Phi_{i}\right\}$ by

$$
\Phi(P)=C_{0}+C_{1} P
$$

the zero point is found at

$$
P_{c}=-C_{0} / C_{1}
$$

The coefficients $C_{0}$ and $C_{1}$ are obtained from

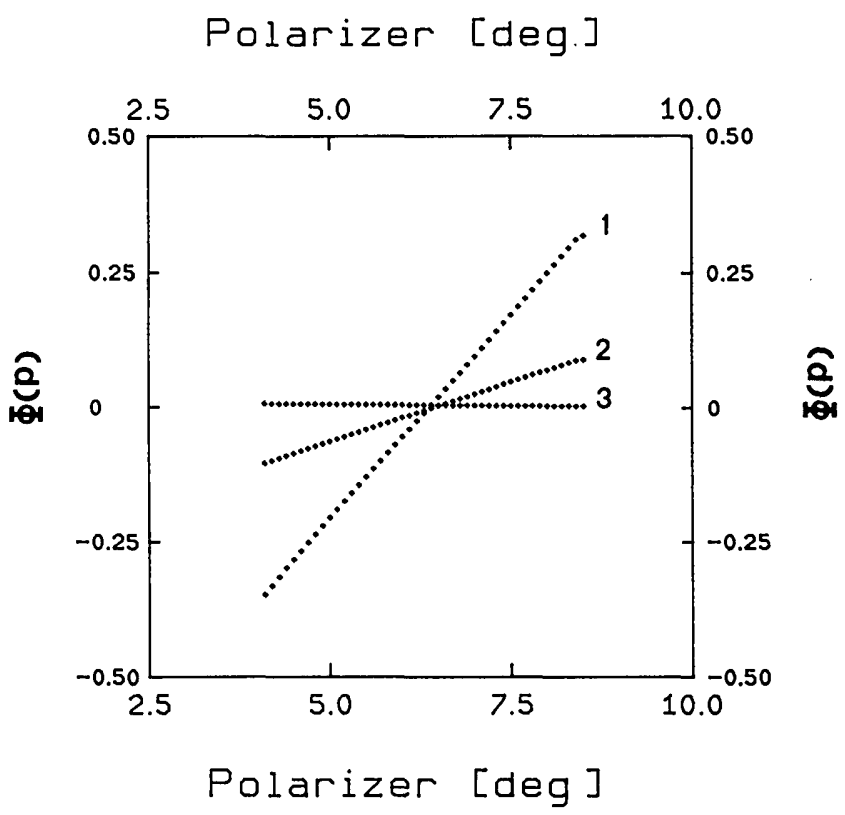

Fig. 4. Three typical examples of phase calibration, where we have plotted the measured phase differences $\Phi(P)$ as a function of the polarizer azimuth $P$. Calibrations 1 and 2 are performed on the same clean $c$-Si sample, calibration 3 on a stainless-steel sample. More information on these calibrations can be found in Table 1 .

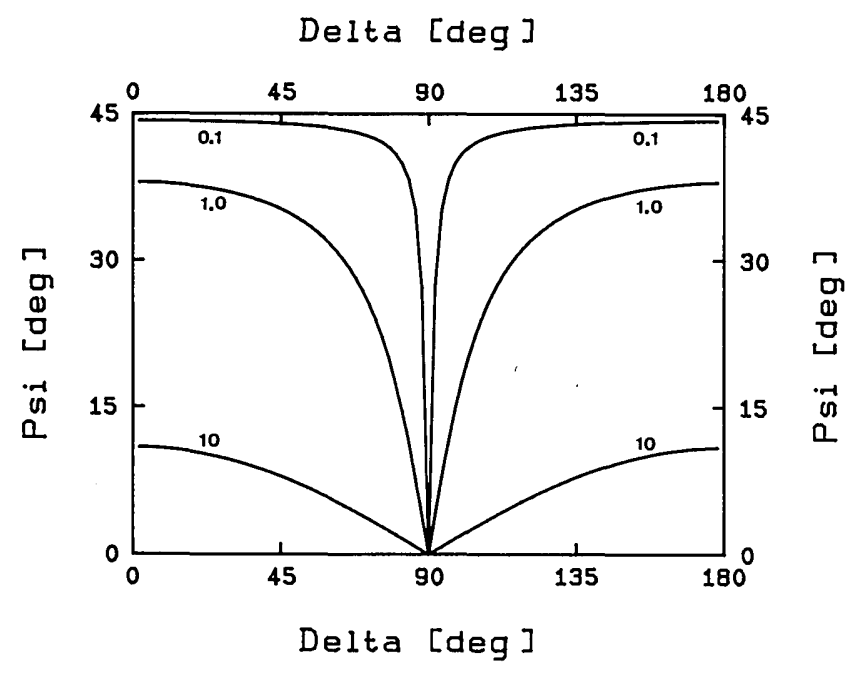

Fig. 5. Curves of constant slope $C_{1}$ of the phase difference $\Phi(P)$ depicted in the $(\Delta, \Psi)$ plane. This method can be applied when $\left|C_{1}\right|$ $>1$.

$$
\begin{aligned}
& C_{1}=(N D)^{-1} \sum_{i=1}^{N}\left(P_{i}-p_{1}\right) \Phi_{i} \\
& C_{0}=N^{-1} \sum_{i=1}^{N} \Phi_{i}-C_{1} p_{1}
\end{aligned}
$$

with $p_{k}$ defined by Eq. (12d) and $D$ redefined:

$$
D=p_{2}-p_{1}{ }^{2} \text {. }
$$

We have repeated the same three calibrations that we presented in Section 3, but now we have used the technique of phase calibration instead of residue calibration. The results of these calibrations can also be found in Table 1 . The table shows that phase calibration works well in the cases of the clean $c$-Si samples, where the measured phase differences $\Phi(P)$ clearly cut the line $\Phi \equiv 0$ (Fig. 4), but that it is of no use for calibrating the stainless-steel sample (calibration 3 ).

We could proceed by discussing the standard deviation of the finally obtained value of $P_{c}$. This property, however, is small under all circumstances; this is clearly shown in Fig. 4. None of these calibrations shows any sign of a statistical error. This lack of statistical error in the phase difference deprives us of a check on the reliability of the calibration as it is seen for the residue calibration. Since the statistical errors are negligible, the systematic errors caused by the component imperfections determine the final error in the calibration. An accurate phase calibration thus requires a phase effect, dominating the possible effects caused by component imperfections, which can be translated to a minimum slope $C_{1}$ for the phase difference as a function of $P$. We have calculated this slope $C_{1}$ from Eqs. (20). The results are depicted in Fig. 5, which shows the lines of constant slope in the $\Delta-\Psi$ plane. A critical value can be obtained from Fig. 4 , assuming that phase calibration 2 is still acceptable, an assumption corresponding to the existence of a critical slope $C_{1} \approx 1$. Inspecting Fig. 5, one notes that this technique of phase calibration works satisfactorily for $\Delta<\pi / 3 \operatorname{rad}$ or $\Delta\rangle$ $2 \pi / 3 \mathrm{rad}$ and $\Psi<\pi / 6 \mathrm{rad}$, while it becomes superior to residue calibration for $\Delta<\pi / 6$ or $\Delta>5 \pi / 6$. 
In conclusion, we can state that the two methods, residue calibration and phase calibration, are complementary.

\section{DISCUSSION}

In the present paper we have discussed the familiar residue calibration method, deriving a rough criterion for its successful application. We have shown that the method becomes less attractive with $\Delta$ approaching 0 or $\pi \mathrm{rad}$, because then random errors in the residues measured will obstruct the determination of the actual position of the minimum. However, tacitly we have assumed that one knows roughly the location of the plane of incidence, allowing one to choose the range of $P$ in order to have $P_{c}$ in the center. In this case the accuracy can be determined by the curvature of the parabola, and Eq. (16) can be applied to obtain the standard deviation of $P_{c}$, which is a good measure of the accuracy. However, if $P_{c}$ does not lie near the center of the initial choice of the range $P$, the parabola will be asymmetric, or even third-order contributions will perturb the curvature of the residue; this in cases of small $C_{2}$, can introduce large errors. Here, too, calculating $\sigma_{P}$ as prescribed by Eq. (16) will provide us a check: if the third-order contribution becomes perturbing, $\sigma_{R}$ will increase significantly, resulting in a $\sigma_{P}$ larger then usual. This shows that the residue method becomes less useful with diminishing $\sin (\Delta)$. Consequently one desires another, complementary method, which works well in the cases when the residue method does not. Phase calibration is such a method; it is suitable for accomplishing calibration under these circumstances. This meth- od, too, is easily implemented on the controlling computer. The disadvantage of phase calibration is its lack of a direct check on the accuracy of the result. The only assurance of accuracy that one can obtain is by calculating the slope of $\Phi(P)$, which should be at least 1 . In general, phase calibration should be preferred to residue calibration for $\Delta<\pi / 6$ or $\Delta>5 \pi / 6$.

\section{REFERENCES}

1. R. M. A. Azzam and N. M. Bashara, "Analysis of systematic errors in rotating-analyzer ellipsometry," J. Opt. Soc. Am. 64, 1459-1469 (1974).

2. J. M. M. de Nijs and A. van Silfhout, "Systematic and random errors in rotating-analyzer ellipsometry," J. Opt. Soc. Am. A 5, 773-781 (1988).

3. D. E. Aspnes, "Effects of component optical activity in data reduction and calibration of rotating-analyzer ellipsometers," J. Opt. Soc. Am. 64, 812-819 (1974).

4. D. E. Aspnes and A. A. Studna, "High precision scanning ellipsometer," Appl. Opt. 14, 220-228 (1975).

5. P. S. Hauge and F. H. Dill, "Design and operation of ETA, an automated ellipsometer," IBM J. Res. Dev. 17, 472-489 (1973).

6. F. L. McCrackin, E. Passaglia, R. R. Stromberg, and H. L. Steinberg, "Measurement of the thickness and refractive index of very thin films and optical properties of surfaces by ellipsometry," J. Res. Natl. Bur. Stand. Sect. A 67, 363-377 (1963).

7. S. Kawabata, "Improved measurement method in rotating-analyzer ellipsometry," J. Opt. Soc. Am. A 1, 706-710 (1984).

8. A. H. M. Holtslag, "Noble gas ion bombardment on clean silicon surfaces, studied using ellipsometry and desorption," Ph.D. dissertation (Twente University, Enschede, The Netherlands, 1986). 